\title{
Prediction of cabling shape during robotic manipulation
}

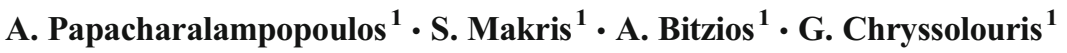

Received: 30 October 2014 / Accepted: 13 May 2015 / Published online: 5 June 2015

(C) The Author(s) 2015. This article is published with open access at Springerlink.com

\begin{abstract}
The manufacturing assembly process includes the manipulation of rigid and non-rigid parts. This paper discusses a method for the estimation of the cables' shape for robotic manipulation. The paper uses methods that take into account the mechanical behaviour of materials. More specifically, in the framework of static analyses, a higher-order analytic model of cables is introduced and the need for model calibration is pointed out. To this effect, analytical solutions are compared against experimental data. In addition, the performance of computational models is taken into consideration.
\end{abstract}

Keywords Flexible parts handling $\cdot$ Harness assembly $\cdot$ Dual arm $\cdot$ Cable shape prediction

\section{Introduction}

Robot-based automation is widely used in automobile manufacturing [1-3]. However, there are some processes that are still carried out manually. The component assembly is a part of such processes. The automated assembly, which needs elaborated movements and a precautious procedure [4], lies in its primary stages of implementation and yields great research interest. When referring to deformable objects $[5,6]$ and cabling, the latter are difficult to be manipulated and predicted in terms of their shape, especially when these parts have a

G. Chryssolouris gchrys@hol.gr

1 Laboratory for Manufacturing Systems and Automation, Department of Mechanical Engineering and Aeronautics, University of Patras, Patras 265 00, Greece complicated form and the strains are exerted dynamically [7-9]. The cases illustrated below are indicative of the problems that industry is faced with, when collision avoidance is desired in cabling manipulation-Fig. 1a, or when motion control in the case of plugging sockets is required-Fig. $1 \mathrm{~b}$.

In order for collisions and other failures to be avoided, a simplified cable model predicting its shape has been suggested. The dynamic behaviour has been approached by a quasi-static process, utilizing different phases of the cable, under the constraint that the robotic manipulator travels in a relatively smooth way. Recent studies on path planning for assembling harnesses have been carried out $[10,11]$ in the automotive industry. The Cosserat rods have been used for the modelling of the harness which is a structure made of complex cables. In a wire harness, there may be a component containing multiple electrical wires with connectors, which have to be attached to the various points of a circular rod. Kyongmo Koo et al. [12] developed an automatic assembly system using three manipulators of seven degrees of freedom each. The harness has clamps and their covers with visual markers attached. The three manipulators visually recognize these markers and cooperate with them. A mass-springdashpot model is used as a dynamic model of deformable linear objects. In these models, the cable is composed of a finite number of nodes. The latter are considered as joints, in order to be able to make a rotation around the three axes. The center of the mass lies at the spherical position joint $i$ and it has a mass $m_{i}$. These joints are connected to rotational springs, which are massless and linear. Rope dynamics are discussed in [13], although the case of the catenary curve may be simplistic.

Analytical methods [14] can be used to describe the bending and twisting states, although the functions are not adequate enough to describe the shape of a cable.

Quasi-static Cosserat media and Kirchoff models [15, 16] are used for predicting the mechanical behaviour of rods. In 
Fig. 1 The concept of automated cabling assembly: collision detection during manipulation (a) and positioning (b). A dual-arm robot is used here and the position of the harness needs to be known in both cases a

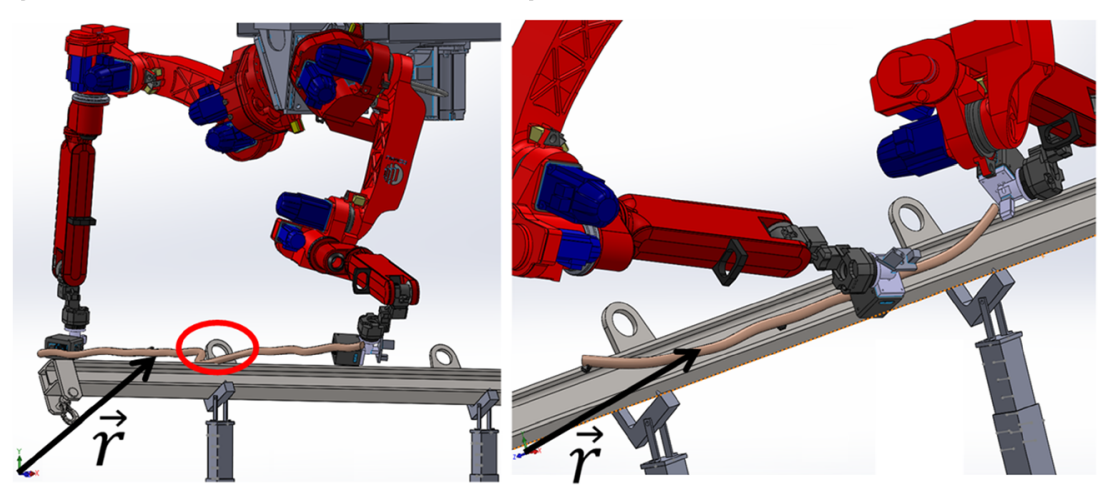

[15], they use a discrete model of an extensible Kirchhoff rod, a special case of Cosserat model theory. In this case, approximate solutions of the equilibrium equations are obtained by means of energy minimization. The latter, as it might be expected, is not exclusively for this theory. It is one of the two methods of obtaining an equilibrium/motion equation. The other one derives from infinitesimal body considerations. In this particular case, the potential energy is discretized and the result is a discrete model of an extensible Kirchhoff rod having a structure similar to that of the "mass and spring model," which can capture the coupling of bending and torsion deformation.

The approach in [17] leads to a more complex form of the kinetic energy. In view of the cable's static solution, a simplified version of gradient elasticity, related to the strain and the strain gradient tensor, is used based on the assumption of a homogeneous and isotropic behaviour. The basic consideration of Mindlin's elastic theory that describes the linear elastic behaviour of isotropic materials with microstructural effects [18-20] is that the potential energy density is a quadratic form of strains, of a gradient of strains, whilst the kinetic energy density is a quadratic form of both velocities and a gradient of velocities. Nevertheless, simplified versions of this theory have been proposed, as 16 intrinsic constants are required this way. Therefore, in the second form of Mindlin's gradient elastic theory, in the potential energy density, among the classical terms, the second gradient of strains is also included.

It is the analytical solution in the simplified strain gradient elasticity theory that is used in the current work.

\section{Cable equilibrium: shape prediction}

\subsection{Comments on models}

Typically, a cable can be deformed under its own weight whilst ideal cables cannot accept any moment. In this work, the cable is being assumed as a continuum [21]. Depending on the loading condition, the equilibrium is used in order for the corresponding differential equation to be obtained. Next, the analytical solutions are used in order for the inadequacies of certain models to be pointed out. Cables in general are described by a second-order differential equation [22] of horizontal direction ( $x$ in Fig. 2). Moments cannot be accepted by the structure.
Fig. 2 Grasping a simple cable (and the physical case)

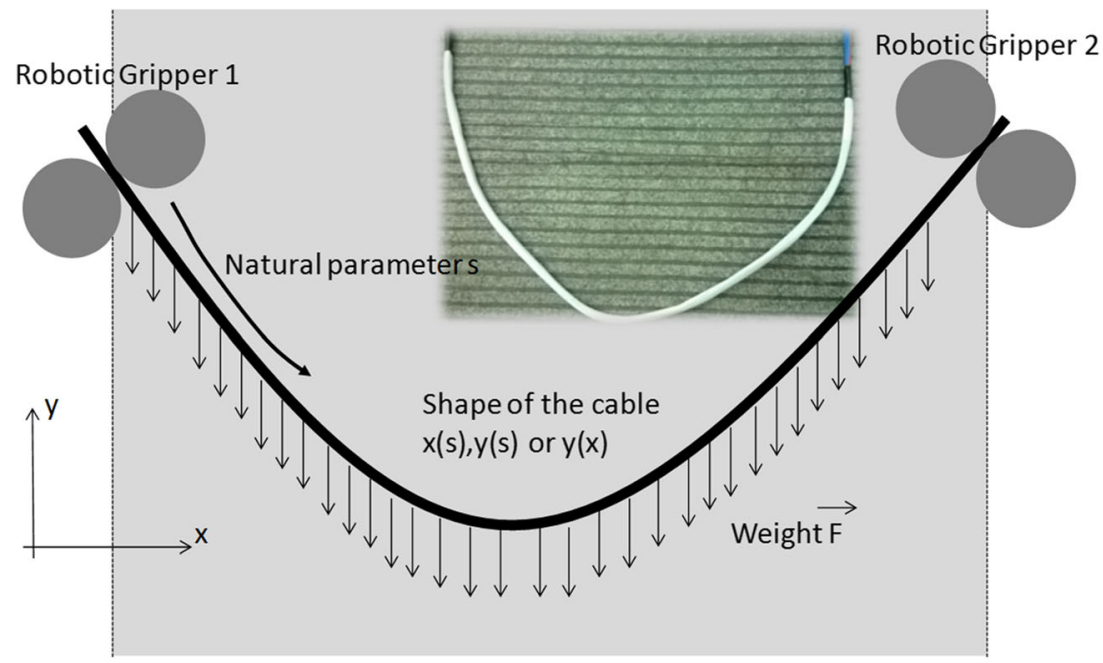




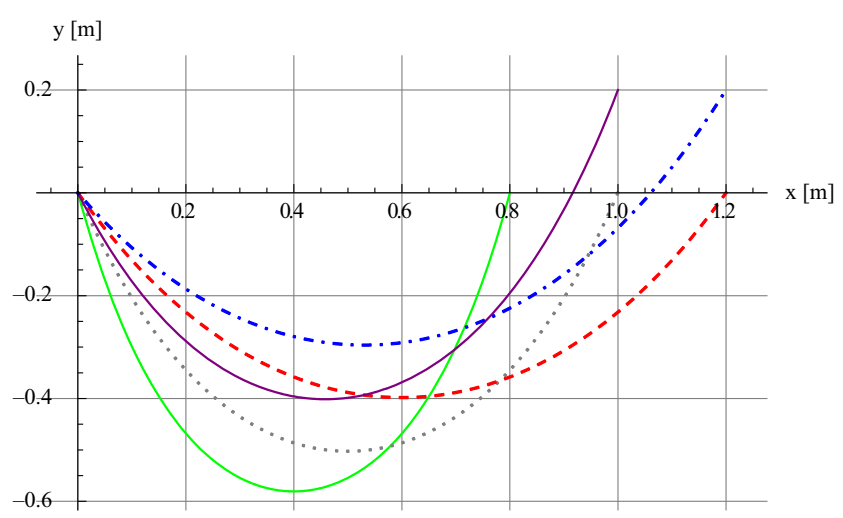

Fig. 3 Simple cable shape prediction (catenary curve) for various positions of robotic grasping point 2

Considering a cable accepting moments, own weight applied, and a non-constant distributed load, depending on $x$, the appropriate general differential equation is the following, utilizing the second and fourth derivatives:

$H y^{\prime \prime}(x)+\beta y^{(4)}(x)=$ loads

with $x$ being the horizontal dimension and $y$ the deflection of the cable. The constant $\beta$, depending on the model's nature, appears in the bibliography, in different versions; in the Timoshenko beam (i.e. [23]), it has to do with bending, and in cases of higher-order theory, such as Mindlin's gradient elasticity [19], it deals with size effects (microstructure affecting macro-behaviour).

Note: The parameter $H$, which is the horizontal component of the cables' tension according to [22], is a free parameter to be chosen in order for the cable's length to equal its physical length, $L$. Solving Eq. 1 analytically for the various values of $H$, it is found that the solution obtained has differences in the corresponding shape. The extra condition that could be used to estimate $H$ is

$$
\int \sqrt{1+y^{\prime 2}} d x=L
$$

Note: Distributed loads are functions of the $x$ coordinate indicating forces acting on the harness. Moreover, concentrated loads, in the case of a harness, can only be added with the help of the Dirac delta distribution, as $\delta(x-w)$. However, $w$, the position in $x$ of the load can only be estimated approximately, as the condition is $\int_{0}^{w} \sqrt{1+y^{\prime 2}} d x=L$, where $w$ is its location on a perfectly stretched cable. This results in a complicated (coupled) form system, as the position of the forces is a function of the cable's shape, comprising the system's solution.

\subsection{Prediction results in an industrial case}

In Fig. 2, a simple, non-extensible cable grasped at both ends is depicted. The objective is that the shape of the cable be predicted. The solution giving the shape within the grasping points (denoted in grey in Fig. 2) is an "interior" solution, whilst the shape of the remaining cable, (usually) hanging on the outside of this area, can be described as an "exterior" solution. The difference between these two lies in the boundary conditions (fixed end vs. free end). The solution to this is well defined namely the catenary curve and it has only one free parameter, driven by the length of the cable in between the grasping points. Various shapes are given in Fig. 3, depending on the position of the second grasping point. The extra condition with reference to the length has been numerically solved with a Gaussian quadrature approximation of 5 points followed by a minimization that was performed, having taken into consideration that the free parameter has to be positive. For the numerical solving of integrals, this method has been used in computational studies [19], known to being exact solutions to polynomial functions [24]. Moreover, for extra simplification of the computational complexity, the cable's shape could be represented by a quadratic function, as it is dictated by the classical cable approach.

In Fig. 4, a cable accepting moments has been taken into consideration. This way, either due to microstructure
Fig. 4 Grasping a cable accepting moments and defining inclination at the grasping points

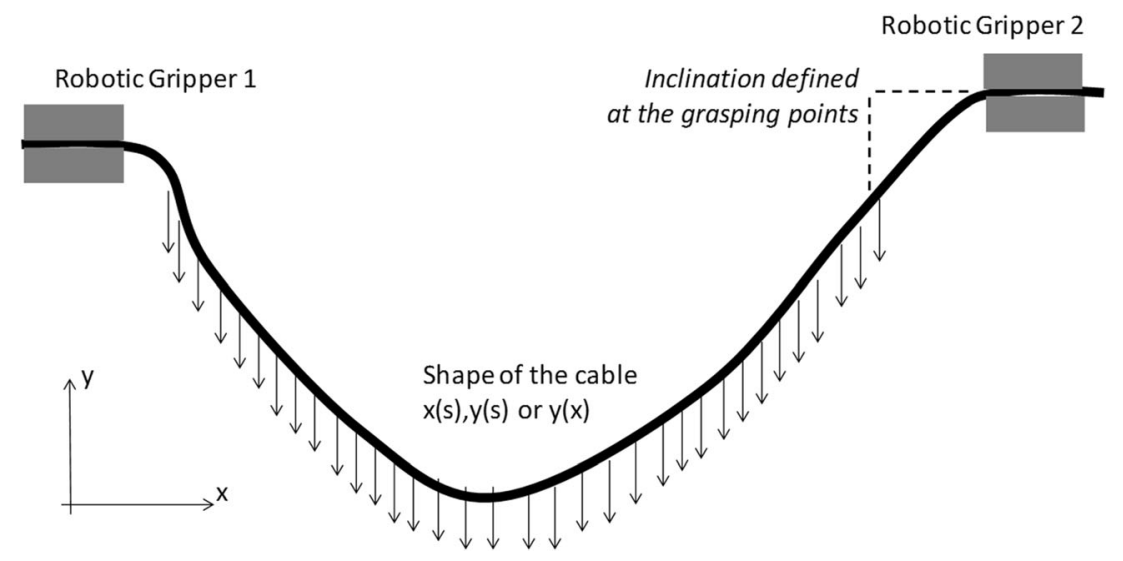




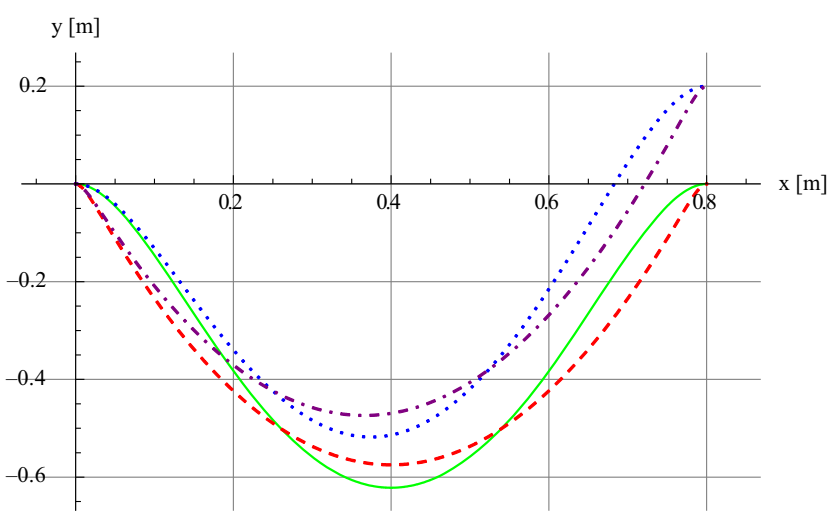

Fig. 5 Alternative cable solution (taking into account bending moments - needs one more free parameter)

or to the Bernoulli-Euler approach, as mentioned above, the inclination is defined at the two ends from the grasping points themselves. The classical model is not good enough for the cable shape prediction, since the boundary conditions are limited.

In Fig. 5, there are some solutions referring to this case (solution of Eq. 3). Two grasping positions have been considered, for each one of the two values of the extra parameter $s$ of Eq. 3. This extra parameter has been proven to be having a limited effect on the shape [19] and, to a great extent, defines the cable's shape near the grasping points, as it is affected by the inclination, found at both ends of the shape. The inclination can be defined since two more boundary conditions are required.

$H y^{\prime \prime}(x)-s y^{(4)}(x)=r x+\beta$

The blue and the black curves correspond to the same boundary conditions, and so do the green and the red ones. Their difference lies in that the inclination, set at the boundaries, affects a greater region of high values of $s$ (green and blue curves), which is an intrinsic parameter of the cable and needs to be calibrated. Higher values of $s$ result in a more stiff mechanical behaviour.

Finally, the solution, out of the grasping points, is taken into consideration. The following figure, Fig. 6, indicates the cable being grasped at one end, on the right, and freed at the other.

This has been solved by minimizing the vertical position of the free end, whilst imposing the length condition. The inclination at the fixed end is set to be horizontal, whilst the inclination at the other end is considered being a minimization output. Its solution is demonstrated in Fig. 7, for various values of the extra parameter $s$, denoting different materials and/or configurations. The blue line indicates a high value for $s$, the green line indicates a lower value, whilst the red line is the solution of the case $s=0$ (classical cable). It is observed that as this parameter approaches zero, the hanging cable tends

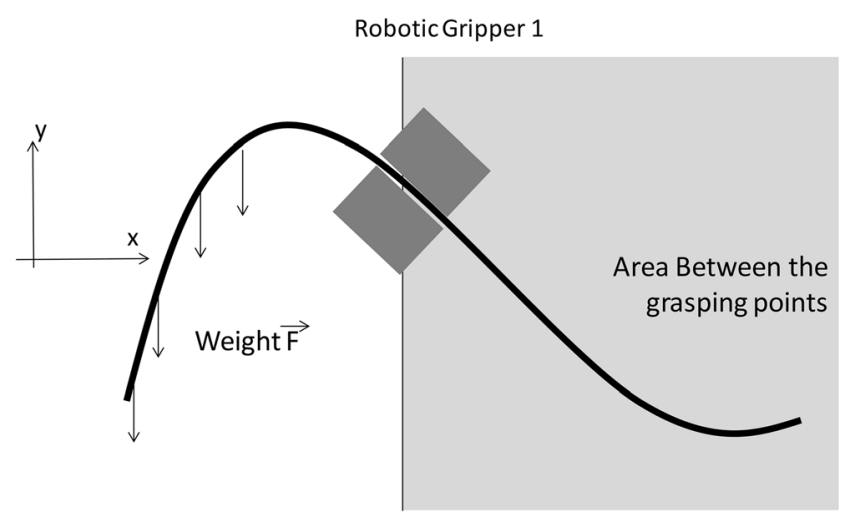

Fig. 6 Simple cable grasping detail

to be a vertical straight line, the result of a cable not accepting moments.

\section{A complex industrial case}

\subsection{Harness analytical model calibration}

The above case referred to a simple cabling system, without too many implications. However, a real harness is much more complicated and it can even comprise a multi-body system. Herein, such a cabling system is captured in two different positions, and a trial to approaching its shape has been made. The equation used and analytically solved is Eq. 3. In this equation, there is a linear distributed load. $H$ is the horizontal component of the cable's tension, $r$ and $\beta$ denote the intensity of the distributed load, whilst $\delta\left(x-k_{i}\right)$ (Dirac delta function) could be used to represent a concentrated load, applied to the $(x-$ $k_{i}$ ) point. The $H$ value was estimated in order for the cable's shape to be as close to the real photo as possible, whilst the value of the $g$ parameter (characteristic microstructure length, estimations of which can be found in [25]) in $s=\mathrm{Hg}^{2}$ was $g=\sqrt{\frac{s}{H}}=8 \mathrm{~cm}$. It is an effective value, meaning that it can be affected by many factors such as thickness and microstructure. One more aspect that could affect this value is the covering of the harness (duct tape like), a pattern of which can be found in detail in Fig. 13(b). The results are shown in Figs. 8 and 9, for the two different postures of the harness. The red line is the $2 \mathrm{D}$ analytical solution, whilst the grid has been digitally added, representing a grid $(10 \times 10 \mathrm{~cm})$. To compare it to a classical solution, that in green, an indicative catenary curve has been added.

The results of the simulation are obviously worse in the second case, and this is potentially due to local twisting (because of the hanging cables) and/or the plastic crease appearing in such cases of deformations (also indicated in Fig. 13(b)). These effects have been deliberately left out of the equation because of their ambiguity. 
Fig. 7 Simple cable grasping detail solution

Fig. 8 Equilibrium of a harness - posture 1. Red line: gradient cable, green line: catenary $\mathrm{y}[\mathrm{m}]$
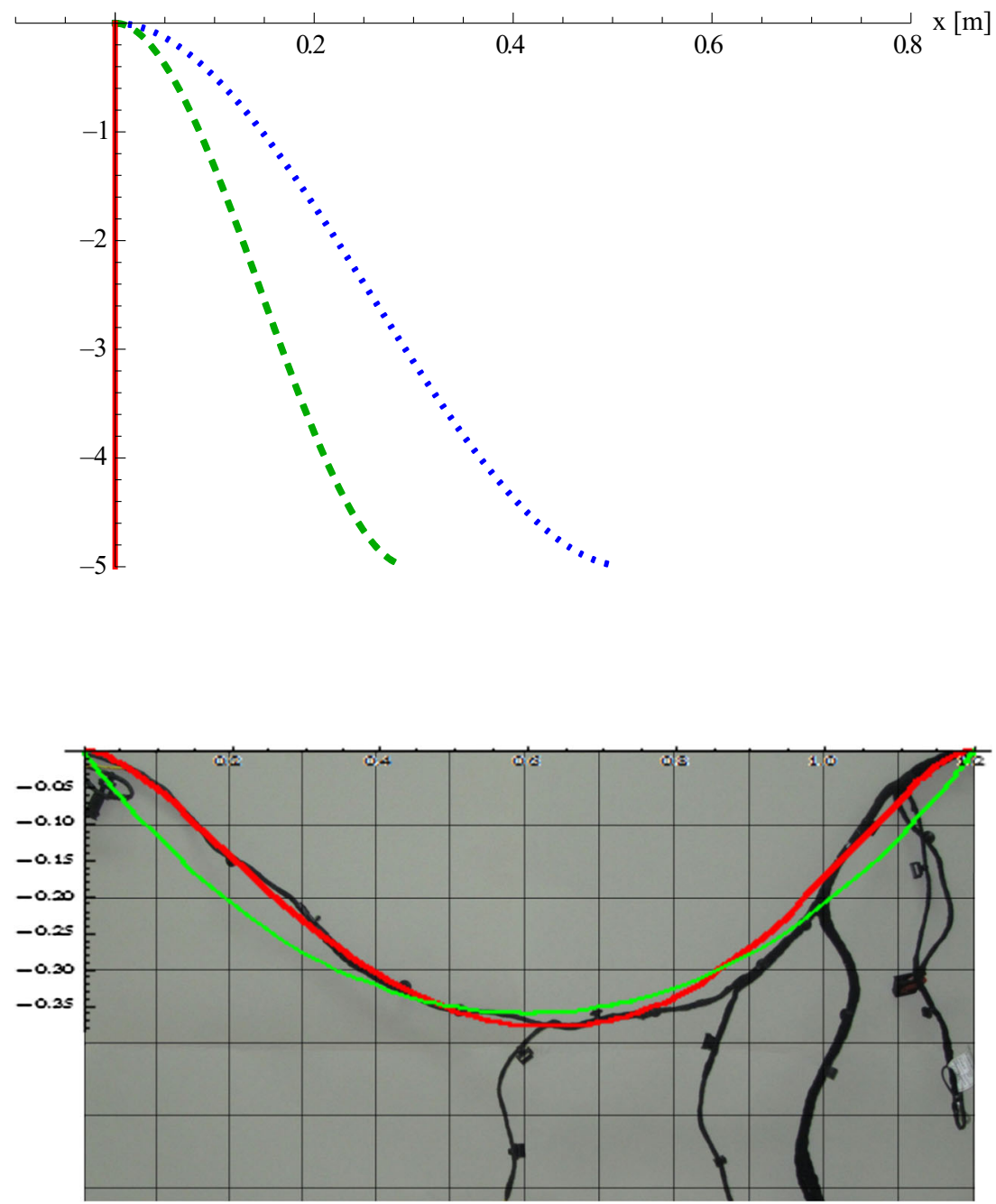

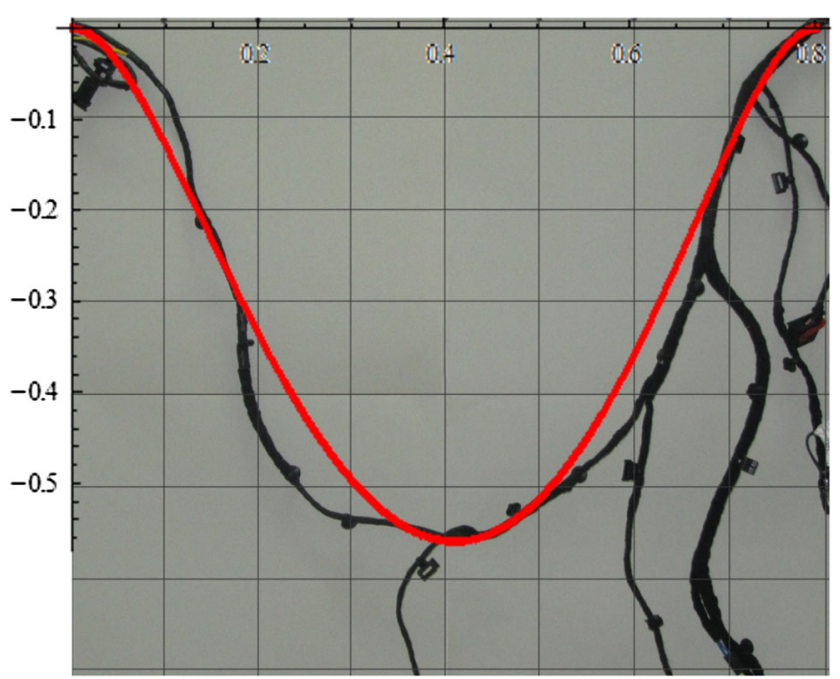

Fig. 9 Equilibrium of a harness - posture 2

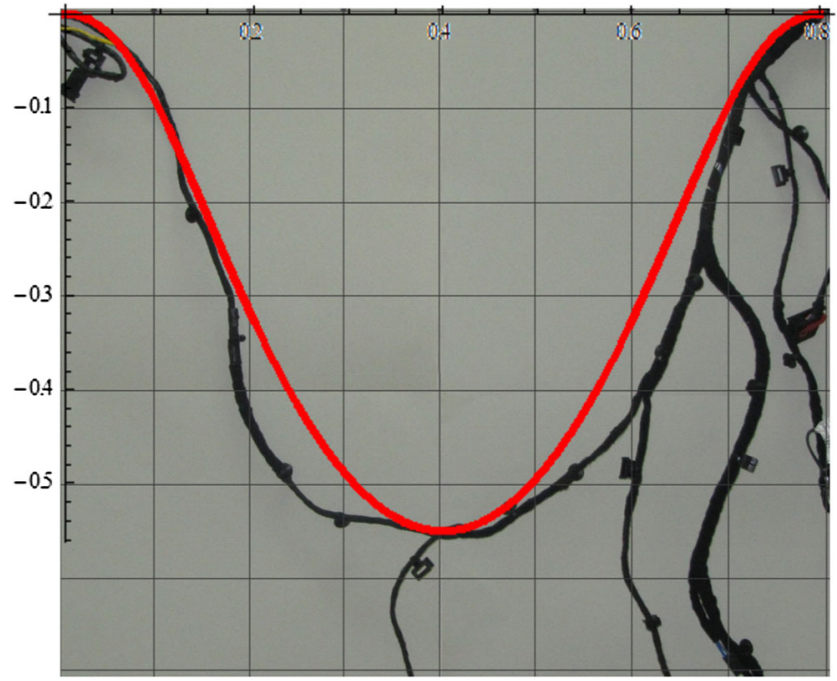

Fig. 10 Equilibrium of a harness - posture 2 - parametric model 


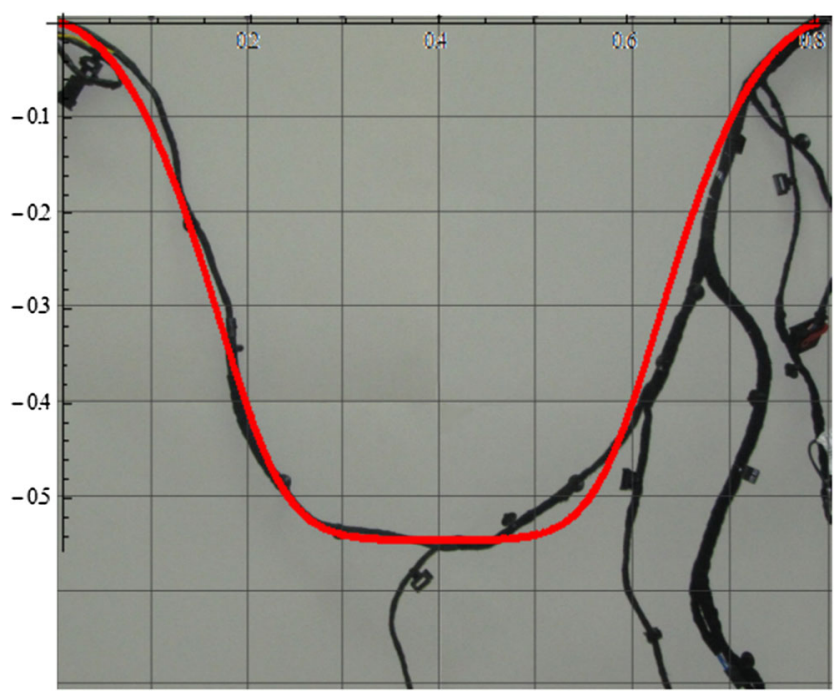

Fig. 11 Equilibrium of a harness - posture 2-parametric model with curvature term

\subsection{Introduction of higher-order terms in parametric modelling}

The physical parameter $l$, which measures the length of the cable ( $l=0$ for one grasping point and $l=L$ for the other, with $L$ being the physical length of the cable), has been taken into account. This leads to acquiring the parametric equations, which enable the formulation of their own weight and the prediction of more complex geometries that cannot be described through a simple function. These equations are

$x^{\prime \prime}(l)-S_{x} x^{(4)}(l)=0$

$y^{\prime \prime}(l)-s_{y} y^{(4)}(l)=w$

The extra parameter has been encapsulated in $w$. Taking into account their analytical equations, the shape prediction result in the case of Fig. 9 can be seen below in Fig. 10. The prediction at the two ends is more justified.
Fig. 12 Equilibrium of a harness - posture 1-parametric model with curvature term
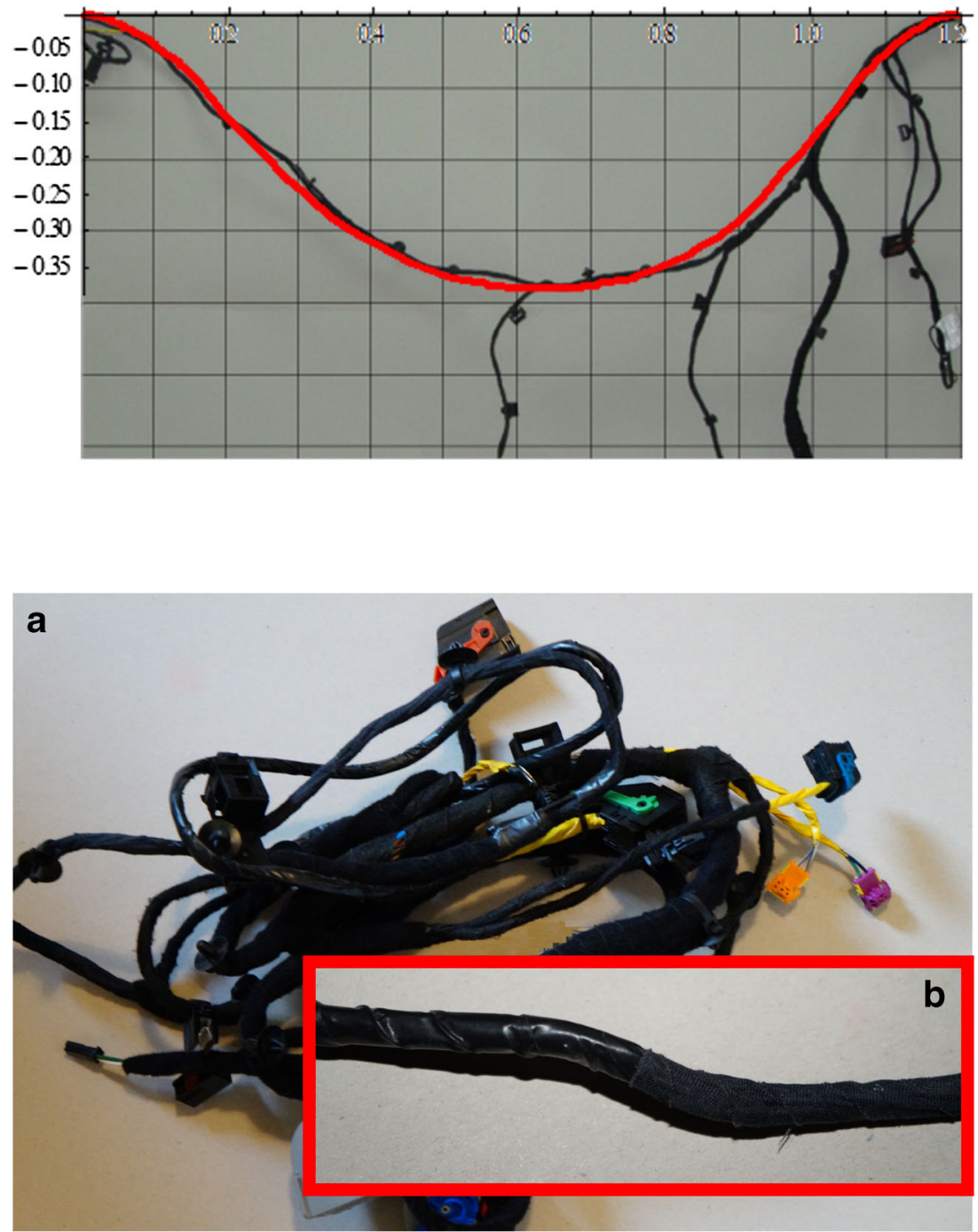

Fig. 13 Picture after folding the cable $(a)$ and detail after stretching the cable $(b)$ 
Fig. 14 Equilibrium of a harness - posture 3-parametric model

Fig. 15 Equilibrium of a harness-posture 3-parametric model with curvature term

Fig. 16 Equilibrium of a

harness - posture 4 - parametric model

Fig. 17 Equilibrium of a harness - posture 4-parametric model with curvature term
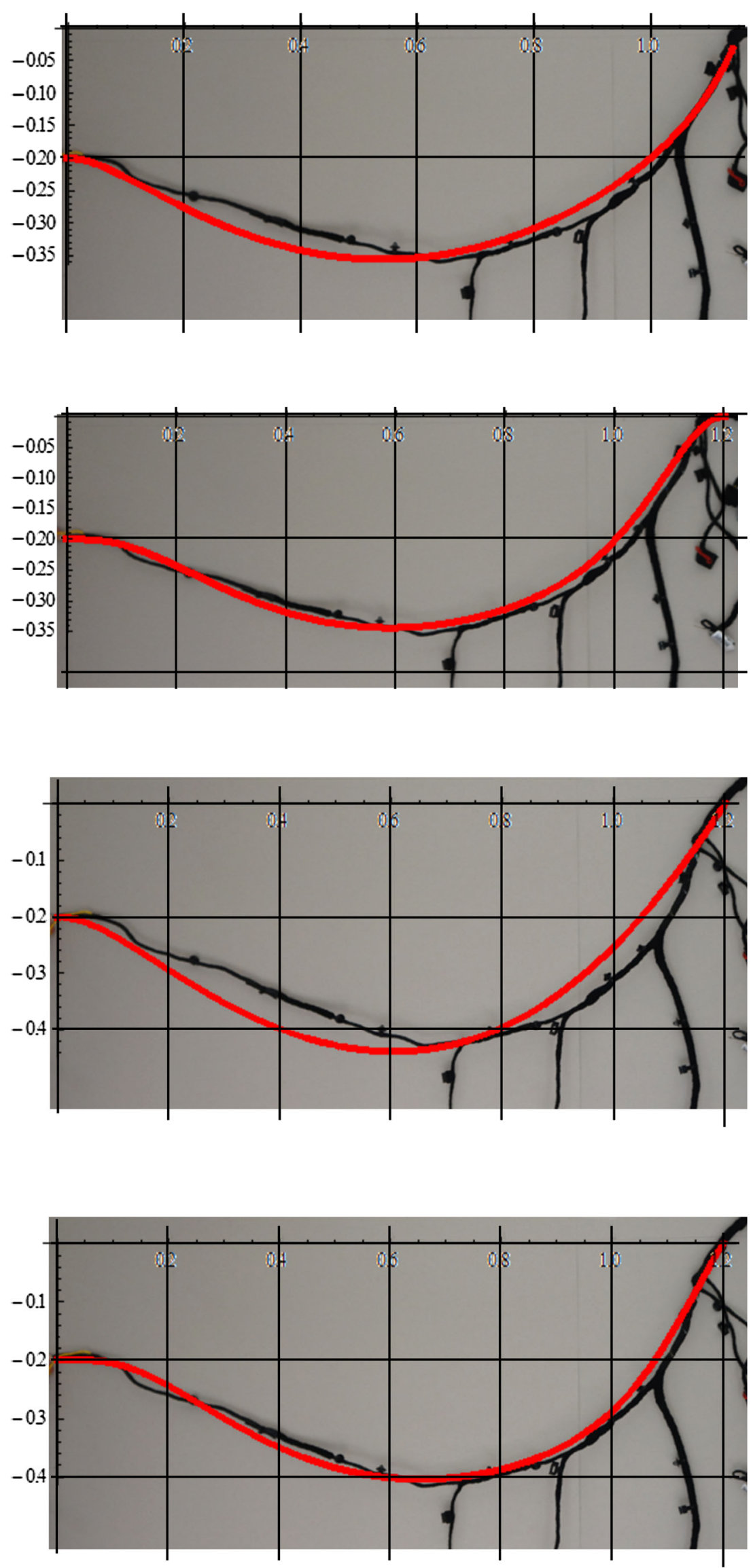
Table 1 Cabling shape prediction error

\begin{tabular}{lllll}
\hline $\begin{array}{l}\text { Posture } \\
\text { ID }\end{array}$ & $\begin{array}{l}\text { Left boundary } \\
\text { conditions }\end{array}$ & $\begin{array}{l}\text { Right boundary } \\
\text { conditions }\end{array}$ & $\begin{array}{l}\text { Prediction error } \\
\text { for the 4th-order } \\
\text { model }\end{array}$ & $\begin{array}{l}\text { Prediction error } \\
\text { for the 6th-order } \\
\text { model }\end{array}$ \\
\hline 1 & $\begin{array}{l}\text { Position }(0,0) \\
\text { Inclination 0 rad }\end{array}$ & $\begin{array}{l}\text { Position }(1.2 \mathrm{~m}, 0) \\
\text { Inclination } 0 \mathrm{rad}\end{array}$ & $2.4 \%$ & $1.3 \%$ \\
2 & $\begin{array}{l}\text { Position }(0,0) \\
\text { Inclination } 0 \mathrm{rad}\end{array}$ & $\begin{array}{l}\text { Inclination } 0 \mathrm{rad} \\
\text { Position }(0.8 \mathrm{~m}, 0)\end{array}$ & $2.2 \%$ & $1.3 \%$ \\
4 & $\begin{array}{l}\text { Position }(0,-0.2 \mathrm{~m}) \\
\text { Inclination 0 rad } \\
\text { Position }(0,-0.2 \mathrm{~m})\end{array}$ & $\begin{array}{l}\text { Inclination } 0 \mathrm{rad} \\
\text { Position }(1.2 \mathrm{~m}, 0)\end{array}$ & $2.3 \%$ & $1.5 \%$ \\
Inclination $0 \mathrm{rad}$ & Inclination $\pi / 4 \mathrm{rad}$ positive & $2.8 \%$ & $1.5 \%$ \\
\hline
\end{tabular}

Fig. 18 Implementation in a simulation package

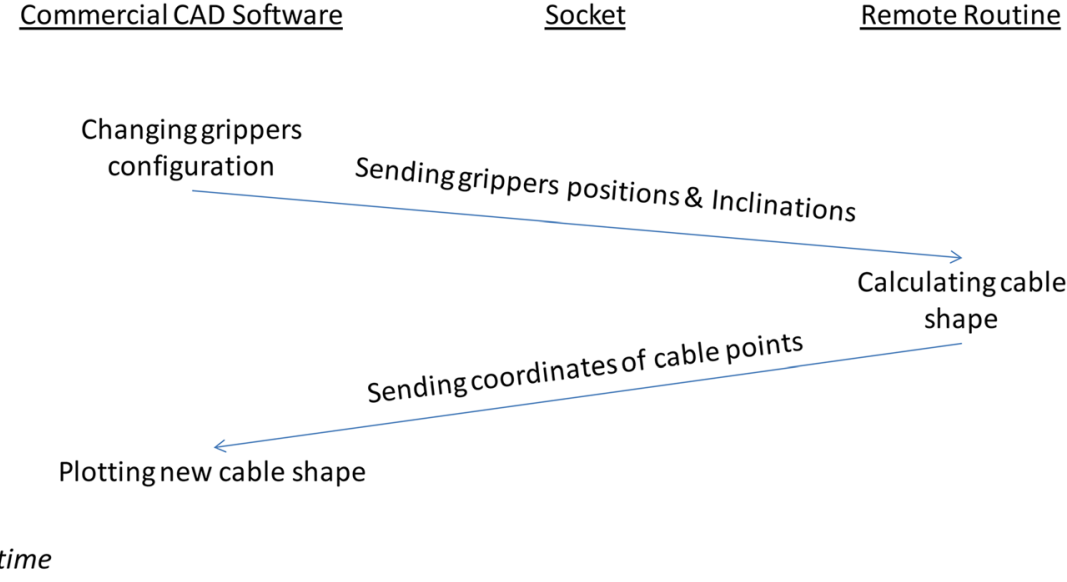

The intuitive relationship between the bending moments (strictly excluding twisting moments, though) and the higher-order derivatives can be shown in equations of pure bending of circular cross-section rods [22], where the parametric equations are given and a higher-than-second-order derivative is included in the equations. The traditional cable model, on the other hand, even in the parametric form, as shown in [26] (simplified version of Eqs. 4 and 5), is strictly of the second order. The fourth-order derivative model, having been introduced at this point for cables of complex structure and originally proposed for elastic structures [27], is a model that predicts moment acceptance and requires two more boundary conditions, namely those of the inclinations at each end. The derivation of this equilibrium (or equivalently motion) equation can come from adding another quadratic term of the second order to the elastic energy [19, 27]. Expanding this methodology, it comes natural to add one more term thus, resulting in the sixth-order equation. As proven below, this allows a better shape prediction. Even more complex models can be suggested, either in terms of the derivative order or that allowing the existence of coupling terms in the equations (or cross partial derivatives). This, however, would require even more calibration experiments, since extra parameters would be required. Moreover, it is imperative that the model require this way far more computational time, resulting in a less efficient simulation. The physical interpretation of the terms and the boundary conditions would also be a major drawback.

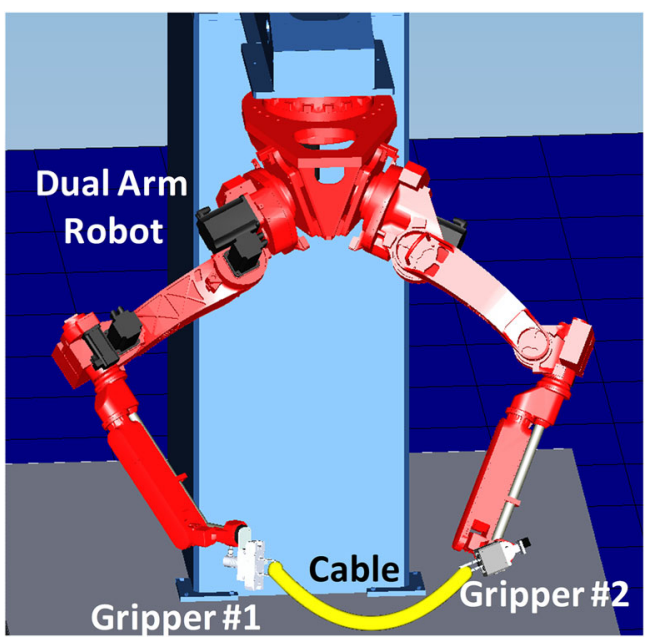

Fig. 19 Screenshot from commercial software 
So, by adding a sixth-order derivative term in Eqs. 4 and 5, the curvature is affected and the following shape prediction (Fig. 11) can be obtained for certain values of the constants.

Finally, in Fig. 12, the same model is proved to be able to predict the first posture of the cable, simply by changing the free $H$ parameter and the second-order derivative boundary condition.

The interpretation of the changing curvature-related boundary condition is that the hanging pieces of the cables deform the curvature of the cable's main body locally in a different way, depending on the angle formed at their connection point. In order for the modelling restrictions and limitations to be studied, the cable is folded as shown in Fig. 13(a) and unfolded. This results in having different crease configurations (one of them is shown in detail in Fig. 13(b)).

The prediction of two more postures is then attempted in terms of shape, as shown in the following figures. Posture 3 regards lowering one end of the cable; Fig. 14 shows a prediction using a model of the fourth order, whilst Fig. 15 illustrates the sixth-order model outcome. Posture 4 is different in terms of inclination as boundary conditions, since the orientation of one gripper changes. The outcomes of the models predicting the shape are given in Figs. 16 and 17, for a fourth- and a sixth-order model, respectively.

The models are characterized by a shape prediction error. This has been defined as the mean distance of the predicted curve from the actual physical cable position, normalized by the total length of the physical cable. The numbers in Table 1 indicate the supremacy (and imply the complexity) of the sixth-order model.

\section{The first step towards implementation}

So far, the solutions refer to a $2 \mathrm{D}$ space. However, for the needs of integrating the model in software, an extension has been made to Eqs. 4 and 5, including also a third component that results in a $3 \mathrm{D}$ system. The shape of the simulation is computed in a remote routine and fed to the robotic simulation software via sockets (Fig. 18).

The time required for the computation of a model is equal to $96 \mathrm{~ms}$ for 150 points on the cable. The result of the simulation, along with rendering a dual-arm robot cell that handles the cable by utilizing two grippers, can be seen in Fig. 19. The cable has been represented as a multi-body system, with the positions of the bodies being the coordinates of the cable.

\section{Conclusions and outlook}

The automated assembly of cables utilizing dual-arm robots requires models that can accurately predict, in a reasonable amount of time, the shape of a manipulated cable. For this reason, an analytical solution to a model of order greater than two has been used. Its validity was checked against the photos of a real cable. Furthermore, the model's suitability was checked against two different postures, in order to check if the parameters used were constant throughout the changing of ambient characteristics.

In a future study, more sophisticated models including other harness characteristics will be tested on. Furthermore, the use of dynamic mechanical models will enhance the assembly procedure in terms of time, since the pre-assumption of the harness, being at equilibrium, will not have to be taken into consideration.

The next step towards the cable and harness assembly, the collision checking and the cable's interaction with other objects, would then occur.

Acknowledgments The work reported in this paper was partially supported by the project X-act/FoFICT-314355, funded by the European Commission in the 7th Framework Programme.

Open Access This article is distributed under the terms of the Creative Commons Attribution 4.0 International License (http:// creativecommons.org/licenses/by/4.0/), which permits unrestricted use, distribution, and reproduction in any medium, provided you give appropriate credit to the original author(s) and the source, provide a link to the Creative Commons license, and indicate if changes were made.

\section{References}

1. Chryssolouris G (2006) Manufacturing systems - theory and practice, 2nd edn. Springer, New York

2. Makris S, Michalos G, Chryssolouris G (2012) Virtual commissioning of an assembly cell with cooperating robots, advances in decision sciences. Volume, Article ID 428060

3. Papakostas N, Michalos G, Makris S, Zouzias D, Chryssolouris G (2011) Industrial applications with cooperating robots for the flexible assembly. Int J Comput Integr Manuf 24(7):650-660

4. Michalos G, Makris S, Rentzos L, Chryssolouris G (2010) Dynamic job rotation for workload balancing in human based assembly systems. CIRP J Manuf Sci Technol 2(3):153-160

5. Ni J, Tang W, Xing Y (2014) Three-dimensional precision analysis with rigid and compliant motions for sheet metal assembly. Int $\mathrm{J}$ Adv Manuf Technol 73:805-819

6. Liu SC, Hu SJ (1997) Variation simulation for deformable sheet metal assemblies using finite element methods. J Manuf Sci Eng 119(3)

7. Dini G, Santochi M (1992) Automated sequencing and subassembly detection in assembly planning. CIRP Ann Manuf Technol 41(1): $1-4$

8. Fantoni G, Santochi M, Dini G, Trach K, Scholz-Reiter BJ, Lien TK, Seliger G, Reinhart G, Franke ., Nørgaard Hansen H, Verl A (2014) Grasping devices and methods in automated production processes. CIRP Ann Manuf Technol 63

9. Hu SJ, Ko J, Weyand L, ElMaraghy HA, Lien TK, Koren Y, Bley H, Chryssolouris G, Nasr N, Shpitalni S (2011) Assembly system design and operations for product variety. CIRP Ann Manuf Technol 60(2):715-733 
10. Hermansson T, Bohlin R, Carlson JS, Söderberg R (2013) Automatic assembly path planning for wiring harness installations. J Manuf Syst 32:417-422

11. Carlson JS, Spensieri D, Söderberg R, Bohlin R, Lindkvist L (2013) Non-nominal path planning for robust robotic assembly. J Manuf Syst 32:429-435

12. Koo K, Jiang X, Konno A, Uchiyama M (2011) Development of a wire harness assembly motion planner for redundant multiple manipulators. J Rob Mechatronics 23(6):907-918

13. Barzel R (1997) Faking dynamics of ropes and springs. IEEE Comput Graph Appl 17(3):31-39

14. Wakamatsu H, Hirai S, Iwata K (1995) Modeling of linear objects considering bend, twist, and extensional deformations. Robot Autom, Proc 1995 I.E. Int Conf, Vol. 1

15. Linn J, Stephan T, Carlsson J, Bohlin (2008) Fast simulation of quasistatic rod deformations for VR applications, progress in industrial mathematics at ECMI 2006. Math Ind 12(2008): $247-253$

16. Linn, J., Stephan, T. (2007), Fast simulation of quasistatic cable deformations using discrete rod models. Multibody Dynamics 2007, ECCOMAS Thematic Conference, Milano, Italy

17. Giannakopoulos AE, Petridis S, Sophianopoulos DS (2012) Dipolar gradient elasticity of cables. Int J Solids Struct 49(10): 1259-1265
18. Papacharalampopoulos A, Stavropoulos P, Doukas C, Foteinopoulos P, Chryssolouris G (2013) Acoustic emission signal through turning tools: a computational study. (CIRP CMMO) Procedia CIRP, 14th CIRP Conference on Modelling of Machining Operations, Turin, 13-14 June

19. Papacharalampopoulos A, Karlis GF, Charalambopoulos A, Polyzos D (2010) BEM solutions for 2D and 3D dynamic problems in Mindlin's strain gradient theory of elasticity. CMES 1622(1):1-29

20. Papacharalampopoulos A, Vavva MG, Protopappas VC, Fotiadis DI, Polyzos D (2011) A numerical study on the propagation of Rayleigh and guided waves in cortical bone according to Mindlin's form II gradient elastic theory. J Acoust Soc Am 130:1060

21. Irvine HM (1992) Cable structures, Dover Publications

22. Landau LD, Lifshitz EM (2003-Reprint) Theory of elasticity, Pergamon Press

23. Timoshenko SP (1922) On the transverse vibrations of bars of uniform cross-section, Philos Mag

24. Davis PJ, Rabinowitz P (1984) Methods of numerical integration, Acad. Press

25. Ben-Amoz M (1976) A dynamic theory for composite material. J Appl Math Phys (ZAMP) Vol. 27

26. Papini D (2010) On shape control of cables under vertical static loads, Master's thesis, Lund University

27. Mindlin RD (1964) Micro-structure in linear elasticity. Arch Ration Mech Anal 16(1):51-78 